\title{
Realization of a Cascaded Quantum System: Heralded Absorption of a Single Photon Qubit by a Single-Electron Charged Quantum Dot
}

\author{
Aymeric Delteil, Zhe Sun, Stefan Fält, and Atac Imamoğlu \\ Institute of Quantum Electronics, ETH Zurich, CH-8093 Zurich, Switzerland
}

(Received 24 January 2017; published 25 April 2017)

\begin{abstract}
Photonic losses pose a major limitation for the implementation of a quantum state transfer between nodes of a quantum network. A measurement that heralds a successful transfer without revealing any information about the qubit may alleviate this limitation. Here, we demonstrate the heralded absorption of a single photonic qubit, generated by a single neutral quantum dot, by a single-electron charged quantum dot that is located $5 \mathrm{~m}$ away. The transfer of quantum information to the spin degree of freedom takes place upon the emission of a photon; for a properly chosen or prepared quantum dot, the detection of this photon yields no information about the qubit. We show that this process can be combined with local operations optically performed on the destination node by measuring classical correlations between the absorbed photon color and the final state of the electron spin. Our work suggests alternative avenues for the realization of quantum information protocols based on cascaded quantum systems.
\end{abstract}

DOI: 10.1103/PhysRevLett.118.177401

In the context of quantum communication and distributed quantum computing, the ability to faithfully transfer a quantum state from one node to another is of key importance [1]. In a direct transfer scheme, quantum information would, in principle, be transmitted between nodes using the consecutive emission, propagation, and absorption of single optical photons [2-5]. However, the realization of such a full procedure is most challenging in any physical system, due to inefficient photon collection from single-photon sources into fibers as well as propagation losses. A previous realization of such a transfer using atoms was achieved with low efficiency and without an experimental verification of a successful transfer [6]. On the other hand, a heralding signal, provided, for instance, by the detection of a subsequently emitted photon at a different wavelength, can make the transfer process robust to losses in protocols where multiple attempts are possible. Previous demonstrations of a heralded photon-to-matter state transfer with atoms or nitrogen vacancy centers were implemented using classical light pulses from weak lasers [7-9] instead of true single-photon pulses generated by a single quantum system. A heralded transfer of quantum information from a single flying qubit to a single stationary qubit has so far remained elusive.

In this Letter, we demonstrate the heralded absorption of a single photonic qubit by a single-electron charged quantum dot (QD). Our work constitutes an experimental realization of a cascaded quantum system [10,11], where fluorescence from a source quantum emitter (a neutral QD) is used to drive a second target quantum emitter (a singleelectron charged QD); the detection of a photon from the latter heralds the success of single-photon absorption. Furthermore, we show that the color of the photon generated by the source QD is classically correlated with the spin state of the electron in the target QD upon the completion of the transfer process. The scheme we realize requires that the source and the target QD have energy level diagrams depicted in Fig. 1(a): The neutral exciton fine structure allows for the possibility to generate a photonic color qubit in an arbitrary superposition of two center frequencies $\omega_{\text {blue }}$ and $\omega_{\text {red }}$ [12]. Degeneracy of the diagonal transitions of the target QD, on the other hand, is essential for heralding the absorption of the incoming photonic qubit in state $\alpha\left|\omega_{\text {red }}\right\rangle+\beta\left|\omega_{\text {blue }}\right\rangle$, without revealing any information about the qubit state. Provided that the electron spin is initially prepared in a superposition state $(|\uparrow\rangle+|\downarrow\rangle) / \sqrt{2}$, the absorption of the incoming photon and the subsequent emission of a diagonal photon at frequency $\omega_{\text {diag }}$ projects the electron spin onto the state $\alpha|\uparrow\rangle+\beta|\downarrow\rangle$. By choosing a single-electron charged source $\mathrm{QD}$, this scheme can be modified to obtain a spin-to-spin quantum state transfer or heralded entanglement generation (see Supplemental Material [13]).

Self-assembled QDs are one of the most favorable systems for building efficient quantum links between remote nodes using optical photons $[12,16]$, due to their integrability into microcavities or photonic nanostructures that allow efficient coupling to a single Gaussian mode $[17,18]$ and near-unity quantum efficiency of emission into a zero-phonon line. In this work, we use two identical samples of single self-assembled InGaAs/GaAs QDs embedded in a lossy planar cavity consisting of a bottom thick (28 periods) distributed Bragg mirror (DBR) and a top thin (two periods) DBR. A $p-i-n$ diode structure is grown around the QD layer for charge state control and Stark tuning of the transitions [19]. A $\mathrm{ZnO}$ solid immersion lens is mounted on top of the samples to increase the collection efficiency. The samples are held in two helium bath 


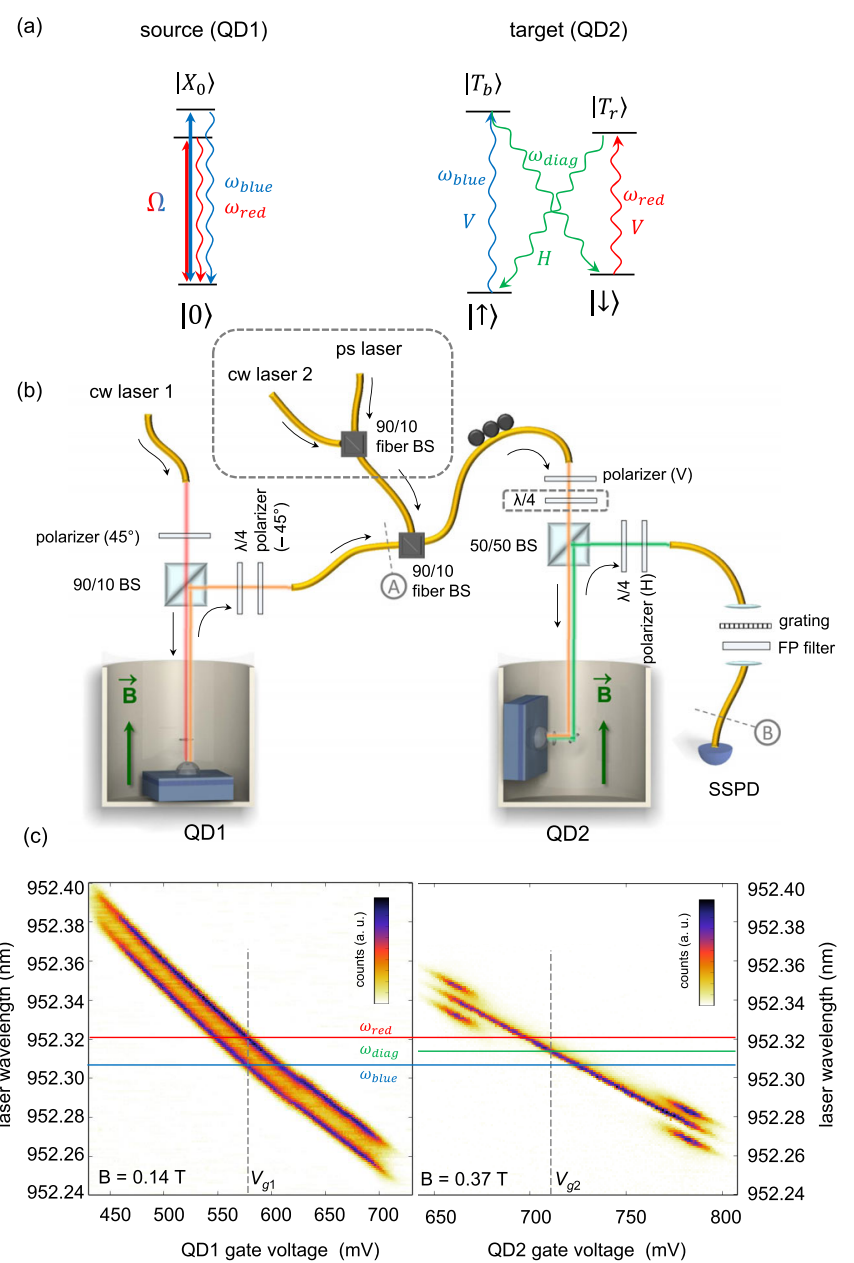

FIG. 1. (a) Energy levels of the source (left) and target (right) QDs. (b) Experimental setup. The neutral exciton transitions of a QD (QD1, left cryostat) are resonantly excited by a two-color laser (cw laser 1). The spontaneously emitted photons are collected into a fiber and conveyed to the input arm of a second cryostat, which hosts a second QD (QD2) in the single-electron charged state, with an external magnetic field applied in the Voigt geometry. The scattered photons are once more collected into a fiber, filtered by a grating and, when applicable, a Fabry-Pérot (FP) filter, and detected with a superconducting single-photon detector (SSPD). The elements in the dashed gray boxes (cw laser 2 , picosecond laser, and quarter-wave plate) are used only in the correlation experiment shown Fig. 3. (b) Resonance fluorescence plateau scans of QD1 and QD2 at the magnetic fields used in the experiments. The vertical axis (laser wavelength) is the same for both plots. The gate voltages used in the experiments is indicated with the vertical dashed line, and the horizontal lines indicate the relevant transition wavelengths.

cryostats with confocal microscopes allowing us to excite QDs with lasers and collect scattered photons through the same objective.

The experimental setup is depicted Fig. 1(b): Singlephoton pulses are generated through the resonant optical excitation of a neutral QD (labeled QD1). By making use of the exciton fine structure, single photons can be generated in any superposition of two center frequencies $\omega_{\text {blue }}$ and $\omega_{\text {red }}$ [12]. After the cross-polarization suppression of the reflected laser light [20], the single photons from QD1 are conveyed via a fiber to another QD located $5 \mathrm{~m}$ away (QD2), which is charged with a single electron by proper tuning of the gate voltage. An external magnetic field is applied to QD2 in the Voigt geometry, making all four transitions from the two trion states to the two spin ground states allowed, with equal oscillator strength. Moreover, QD2 has been selected to have its two diagonal transitions almost degenerate, which is ensured by opposite in-plane $g$ factors for the electron and the hole. Although such QDs can be naturally found in self-assembled QD samples (see Supplemental Material [13]), the diagonal transitions can also be tuned to resonance using an external electric field [21], strain [22], ac-Stark shift [23], or magnetic field orientation using a vector magnet.

Figure 1(c) shows resonance fluorescence plateaus of the two QDs. While the QD1 resonance fluorescence plateau exhibits standard fine-structure-split lines of the neutral exciton, the QD2 plateau presents a unique signature of degenerate diagonal transitions: Instead of four resonance fluorescence lines visible only near the edges of the plateau due to spin pumping [24], only three lines are visible. Moreover, the intensity of the intermediate resonance fluorescence line does not vanish in the middle of the gate voltage range, since a laser at $\omega_{\text {diag }}$ ensures that there is no spin pumping. The external electric and magnetic fields are adjusted such that the two transitions of QD1 match the blue and red vertical transitions of QD2, as we indicate in Fig. 1(c). For QD2, we have carried out Hong-Ou-Mandel interferometry to verify the indistinguishability of photons emitted at $\omega_{\text {diag }}$ (see Supplemental Material [13]).

The first part of our demonstration consists in exciting QD1 with a two-color laser in cw (labeled cw laser 1), generated from a single-mode laser using an electro-optic modulator (EOM) driven with a microwave signal at $\left(\omega_{\text {blue }}-\omega_{\text {red }}\right) / 2$. The resonant fluorescence photons are brought to the second cryostat, and their polarization is made vertical $(V)$. The photons that are scattered a second time by QD2 pass through a horizontally $(H)$ oriented polarizer that suppresses reflected QD1 light, such that we collect only diagonal photons at frequency $\omega_{\text {diag. }}$. The collection path also includes a grating of bandwidth $\sim 80 \mathrm{GHz}$ and a flip-mounted Fabry-Pérot filter (that we do not use in this part of the experiment). Finally, we placed a superconducting single-photon detector (SSPD) at the output port of the collection fiber. Its dark count rate is lower than $1 \mathrm{~s}^{-1}$, and so is the background light detection rate, leading to a false event recording rate lower than $2 \mathrm{~s}^{-1}$, which is essential for the experiments presented here.

When we fix the laser wavelength and the gate voltage of QD2 at resonance, the count rate shows a Lorentzian line shape as a function of QD1 gate voltage, with a maximum of about 90 counts per second [Fig. 2(a)]. Fixing QD1 and 

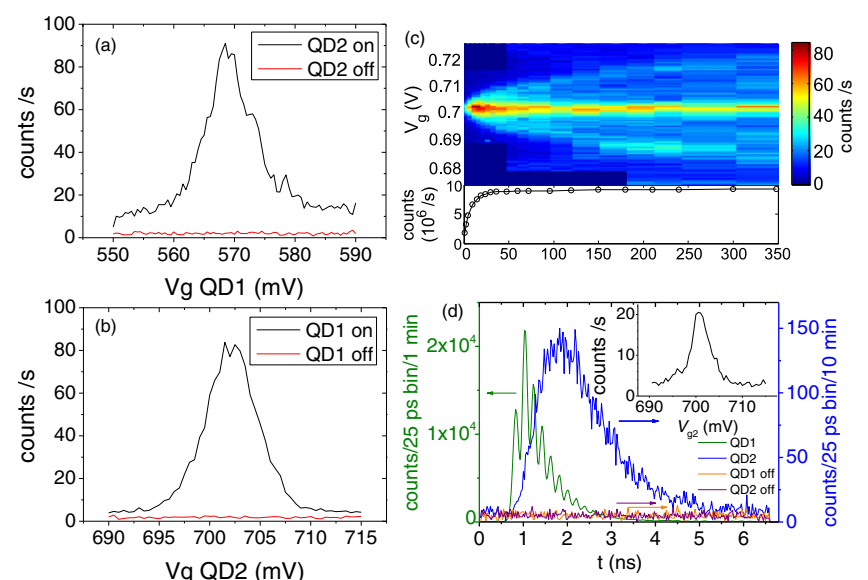

FIG. 2. (a) Photon count rate as a function of the QD1 gate voltage, with QD2 fixed on resonance (black curve) or off resonance (red curve). The integration time is $10 \mathrm{~s}$ per data point. (b) Photon count rate as a function of the QD2 gate voltage, with QD1 fixed on resonance (black curve) or off resonance (red curve). (c) Upper panel: Count rate as a function of the QD1 gate voltage and laser power, revealing the Mollow sidebands at a high power. Lower panel: The corresponding count rate of QD1 measured directly at the output fiber of the left cryostat [point $A$ in Fig. 1(a)], as a function of the laser power. (d) Time-resolved count rate, with a repetition rate of $7 \mathrm{~ns}$. Green curve, without cross-polarization suppression, revealing the single-photon pulse envelope from QD1. Blue curve, with cross-polarization suppression, showing the time-resolved heralded absorption rate. Orange (purple) curve, the same as the blue curve, but when QD1 (QD2) is tuned off resonance. Inset: Average count rate as a function of QD2 detuning.

varying QD2 leads to a similar result [Fig. 2(b)]. When either dot is tuned off resonance, the count rate remains as low as $2 \mathrm{~s}^{-1}$, close to the background value, irrespectively of the gate voltage of the other dot [red curves in Figs. 2(a) and 2(b)], demonstrating unequivocally that we have implemented a heralded single-photon absorption. For these measurements, the laser power is set close to the saturation power of QD1, corresponding to the detection of about $5.5 \times 10^{6}$ counts/s at the output fiber of the first cryostat [point $A$ on Fig. 1(a)]. Although QD1 can be driven at a higher power, leading to a detection rate up to almost 10 million counts/s, it does not result in an increase of the heralded absorption rate. Indeed, when increasing the power above saturation, the photons emitted by QD1 start having a spectral mismatch with QD2 transitions, attributed to the appearance of Mollow sidebands [20,25]. These sidebands can be directly observed by means of the heralded scattering rate as a function of the gate voltage of QD2 as can be seen in Fig. 2(c).

The quantum efficiency of the heralded absorption process can be estimated by knowing the total transmission of our setup [ $0.3 \%$, measured from $A$ to $B$ in Fig. 1(a)] and taking into account the collection efficiency of the scattered photons, including the collection at the first lens $(20 \%$, deduced from the maximum count rate and the measured excited state lifetime) and the polarizer (blocking 50\% of the scattered photons). We deduce that about $8 \%$ of the incident photons are absorbed by QD2. This experiment is done with a random spin population of QD2, leading to an additional reduction of $50 \%$ in this probability - the quantum efficiency is therefore estimated to be about $16 \%$. This number is consistently close to the overlap of QD2 emission with the free-space mode collected by the microscope objective ( $\sim 20 \%)$ which gives the maximum absorption probability of a single incident photon by a single quantum emitter [26].

This experiment can also be performed in the pulsed regime, allowing us to observe the dynamics of the process within a few tens of picoseconds [Fig. 2(d)]. In this case, QD1 is driven by a two-color laser pulse of $400 \mathrm{ps}$ (from cw laser 1), generating two-color single-photon pulses with a repetition rate of $152 \mathrm{MHz}$. They can be directly detected by removing the crossed-polarization suppression [green curve in Fig. 2(d)]. The time-resolved detection rate exhibits a beat note at $\omega_{\text {blue }}-\omega_{\text {red }}=4.9 \mathrm{GHz}$, whose visibility is limited by the jitter of our detector. The $g^{(2)}(0)$ of the single-photon pulses is measured to be $0.15 \pm 0.02$, limited by double excitations during the 400-ps laser pulse. When the crossed polarizer is set, the photons at frequency $\omega_{\text {diag }}$ scattered by QD2 can then be observed [blue curve in Fig. 2(d)]. We also verify once more that the counts vanish when either dot is switched off resonance [orange and purple curves in Fig. 2(d)].

The time-resolved detection of these doubly scattered photons well above the background opens the possibility to implement quantum information protocols based on such cascaded quantum systems, by combining heralded absorption of photonic qubits with local operations such as state initialization, manipulation, and readout. In the following, we demonstrate an experimental implementation of a photon-to-spin state transfer protocol, and we show classical correlations between the photonic qubit state and the final spin state.

The setup for this experiment is the same as depicted in Fig. 1(a) [13], with two additional lasers (labeled cw laser 2 and ps laser) driving QD2. The pulse sequence is shown in Fig. 3(a). The spin state of QD2 is prepared in a superposition of equal weights by spin pumping in $|\uparrow\rangle$ or $|\downarrow\rangle$ followed by a rotation of $\pi / 2$ performed using a 15-ps laser pulse detuned by $1 \mathrm{THz}$ from the trion resonances. This rotation requires the insertion of a quarter-wave plate in the input arm such that the excitation port is elliptically polarized [27]. As a consequence, the crossed-polarized detection path is also elliptically polarized and therefore does not reject all the $V$-polarized blue and red photons scattered by QD2. They are then filtered out by adding a Fabry-Pérot filter in the collection path, having one of the transmission maxima centered at $\omega_{\text {diag. }}$. A single photon pulse, generated by the excitation of QD1 exciton, is coupled to QD2 after a delay time of $\sim 15 \mathrm{~ns}$, chosen such that the trion population has sufficiently decayed to observe 

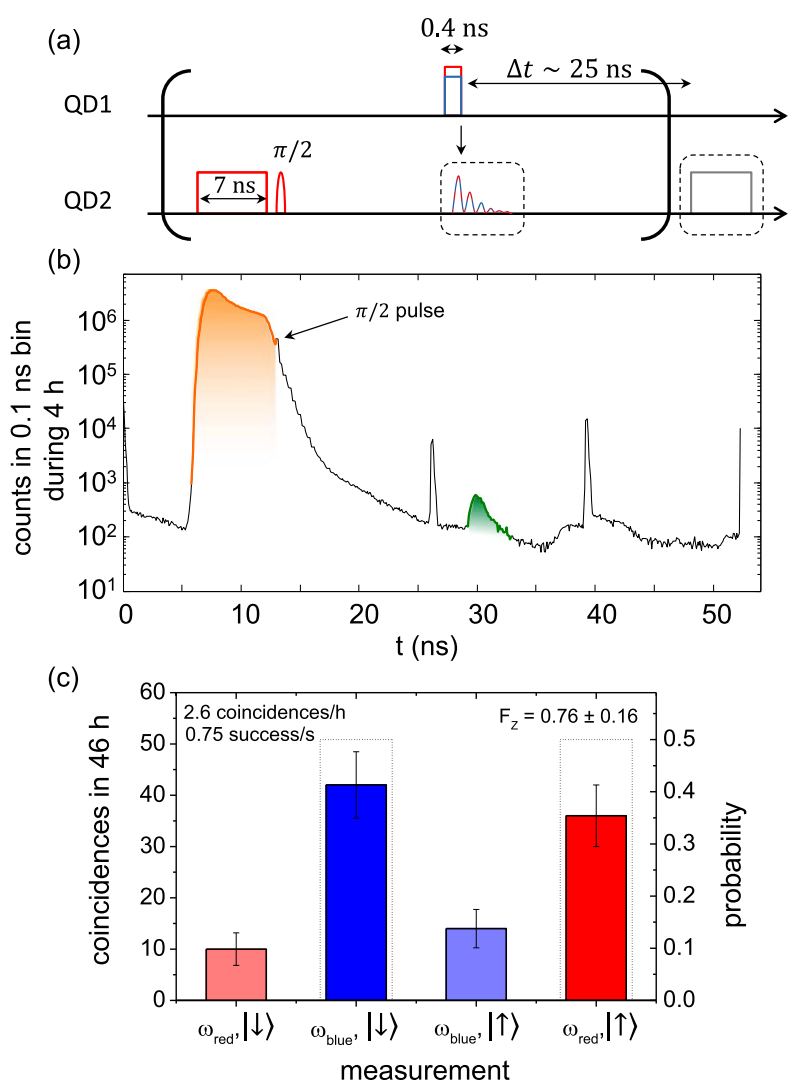

FIG. 3. (a) Pulse sequence used to measure classical correlations. Single-photon pulses are generated from QD1 using a 0.4 ns pulse, resonant with either of the exciton resonances. QD2 is prepared in state $(|\uparrow\rangle+|\downarrow\rangle) / \sqrt{2}$ using spin pumping followed by a $\pi / 2$ rotation. These pulses are combined with the single-photon pulse generated using QD1. (b) Photon count histogram recorded during a 4-h-long measurement. The events involved in the twofold coincidence calculation are shaded in orange (spin measurement) and in green (heralded absorption of a single photon from QD1). The peaks at 26, 39, and $52 \mathrm{~ns}$ are residual picosecond pulses from the rotation laser that are suppressed by $\sim 10^{2}$ using an EOM. (c) Twofold coincidence rate corresponding to a photon detected during the spin pumping pulse conditioned on a photon heralding a single photon absorption in the previous period. The dashed bars correspond to the case of perfect classical correlations.

heralded absorption above the spontaneous emission background. After an additional time of $25 \mathrm{~ns}$ (corresponding to the detector dead time), the pulse sequence is repeated, and the subsequent spin pumping pulse serves to measure the spin state in the computational basis states. As we do not perform dynamical decoupling, the spin superposition precesses with a random frequency during the protocol. Since in this work we demonstrate only classical correlations, this random phase plays no role in the obtained results. A detailed scheme of the experimental setup including the generation and synchronization of the optical pulses is available in Supplemental Material [13].

Figure 3(b) shows a time trace recorded during $4 \mathrm{~h}$. One of the major technical difficulties is to keep a low background-at least in the vicinity of the time window where we detect heralded absorption-when combining strong resonant laser pulses of $P \sim P_{\text {sat }}$ with the singlephoton pulses. As usual, EOMs have finite on/off ratios (typically $10^{2}-10^{3}$ ) leading to a constant resonance fluorescence background of a few thousands of counts per second (for a dot showing a few $10^{6}$ counts per second around saturation); we cascade three EOMs to reach an on/off ratio of $10^{6}$. This, in turn, suppresses the scattered photon background and allows for the observation of heralded absorption. A waiting time of $\sim 15 \mathrm{~ns}$ after the spin pumping or measurement pulse and the optically performed spin rotation allows the trion population we generated to decrease by 4-5 orders of magnitude through radiative decay, since the latter would also lead to detection events hindering our signal. The slower time scale of this biexponential decay is attributed to two-photon absorption during the strong rotation pulse followed by the relaxation to the trion states (see Supplemental Material [13] for more details about contributions to the background).

The four combinations of input photon color (red and blue) and spin state measurement $(|\uparrow\rangle$ and $|\downarrow\rangle)$ are alternated, and classical correlations between the photon color and the spin state are deduced from the twofold coincidences of a first photon detected during the heralded absorption window [green shading in Fig. 3(b)] together with a photon detected during the following spin pumping pulse [orange shading in Fig. 3(b)]. Using a repetition rate of $20 \mathrm{MHz}$, we obtain a success rate (rate of heralded absorption events) of $0.75 \mathrm{~s}^{-1}$, corresponding to the count rate in the green shaded time window in Fig. 3(b). It is smaller than the rate shown in Fig. 2(d) due to the much lower repetition rate as well as the use of elliptic polarization in both input and output arms and the FP filter transmission. This leads to a twofold coincidence rate of 2.6 per hour. The result from data recorded during $46 \mathrm{~h}$ is shown in Fig. 3(c). For a red (blue) input photon, the spin is measured in $|\uparrow\rangle(|\downarrow\rangle) \sim 3.5$ times more often than in $|\downarrow\rangle$ $(|\uparrow\rangle)$, consistent with what is expected [cf. the QD2 level scheme in Fig. 1(a)]. It leads to a computation basis fidelity of $0.76 \pm 0.16$, mainly limited by the background photons during the heralded absorption process.

Our experiments implement a cascaded quantum system where a single photon in a superposition of two color states generated by a quantum emitter drives another quantum emitter with a spin-1/2 ground state [10,11]. While heralding of the photon absorption allows us to measure classical correlations between the photonic input and the output spin states, the current success rate is not high enough to implement a state transfer in a quantum network. If the success rate can be enhanced, then a heralded quantum state transfer can be combined with a quantum error correction. In particular, for a photon loss rate less than $2 \%$ [28], it is possible to use erasure codes to achieve arbitrarily high efficiency in a quantum state transfer. In this 
low-loss limit, the lack of a heralding event for one of the physical photonic qubits would constitute a syndrome measurement indicating an erasure error. Moreover, extensions of this work can also apply to the realization of hybrid quantum networks connecting different physical systems via photons [4].

This work is supported by NCCR QSIT (National Centre of Competence in Research, QSIT-Quantum Science and Technology), the research instrument of the Swiss National Science Foundation (SNSF), and by the Swiss NSF under Grant No. 200020-159196.

[1] D. P. DiVincenzo, Fortschr. Phys. 48, 771 (2000).

[2] J. I. Cirac, P. Zoller, H. J. Kimble, and H. Mabuchi, Phys. Rev. Lett. 78, 3221 (1997).

[3] Y. L. A. Rezus, S. G. Walt, R. Lettow, A. Renn, G. Zumofen, S. Götzinger, and V. Sandoghdar, Phys. Rev. Lett. 108, 093601 (2012).

[4] H. M. Meyer, R. Stockill, M. Steiner, C. Le Gall, C. Matthiesen, E. Clarke, A. Ludwig, J. Reichel, M. Atatüre, and M. Köhl, Phys. Rev. Lett. 114, 123001 (2015).

[5] N. Piro, F. Rohde, C. Schuck, M. Almendros, J. Huwer, J. Ghosh, A. Haase, M. Hennrich, F. Dubin, and J. Eschner, Nat. Photonics 7, 17 (2011).

[6] S. Ritter, C. Nölleke, C. Hahn, A. Reiserer, A. Neuzner, M. Uphoff, M. Mücke, E. Figueroa, J. Bochmann, and G. Rempe, Nature (London) 484, 195 (2012).

[7] C. Kurz, M. Schug, P. Eich, J. Huwer, P. Müller, and J. Eschner, Nat. Commun. 5, 5527 (2014).

[8] N. Kalb, A. Reiserer, S. Ritter, and G. Rempe, Phys. Rev. Lett. 114, 220501 (2015).

[9] S. Yang et al., Nat. Photonics 10, 507 (2016).

[10] H. J. Carmichael, Phys. Rev. Lett. 70, 2273 (1993).

[11] C. W. Gardiner, Phys. Rev. Lett. 70, 2269 (1993).

[12] W.-B. Gao, P. Fallahi, E. Togan, A. Delteil, J. MiguelSanchez, and A. Imamoğlu, Nat. Commun. 4, 2744 (2013).
[13] See Supplemental Material at http://link.aps.org/ supplemental/10.1103/PhysRevLett.118.177401, which includes Refs. [14,15], for more details about the setup and the contributions to the background noise, as well as for more details about QD2 characterization.

[14] W. B. Gao, P. Fallahi, E. Togan, J. Miguel-Sanchez, and A. Imamoğlu, Nature (London) 491, 426 (2012).

[15] T. B. Pittman, B. C. Jacobs, and J. D. Franson, Phys. Rev. Lett. 88, 257902 (2002).

[16] A. Delteil, Z. Sun, W. B. Gao, E. Togan, S. Faelt, and A. Imamoğlu, Nat. Phys. 12, 218 (2015).

[17] O. Gazzano, S. Michaelis de Vasconcellos, C. Arnold, A. Nowak, E. Galopin, I. Sagnes, L. Lanco, A. Lemaître, and P. Senellart, Nat. Commun. 4, 1425 (2013).

[18] M. Munsch, N. S. Malik, E. Dupuy, A. Delga, J. Bleuse, J.-M. Gérard, J. Claudon, N. Gregersen, and J. Mø rk, Phys. Rev. Lett. 110, 177402 (2013).

[19] D. Pinotsi, P. Fallahi, J. Miguel-Sánchez, and A. Imamoğlu, IEEE J. Quantum Electron. 47, 1371 (2011).

[20] A. N. Vamivakas, Y. Zhao, C.-Y. Lu, and M. Atatüre, Nat. Phys. 5, 198 (2009).

[21] J. H. Prechtel, F. Maier, J. Houel, A. V. Kuhlmann, A. Ludwig, A. D. Wieck, D. Loss, and R. J. Warburton, Phys. Rev. B 91, 165304 (2015).

[22] H. M. G. A. Tholen, J. S. Wildmann, A. Rastelli, R. Trotta, C. E. Pryor, E. Zallo, O. G. Schmidt, P. M. Koenraad, and A. Yu. Silov, Phys. Rev. B 94, 245301 (2016).

[23] R. Bose, T. Cai, G. S. Solomon, and E. Waks, Appl. Phys. Lett. 100, 231107 (2012).

[24] X. Xu, Y. Wu, B. Sun, Q. Huang, J. Cheng, D. G. Steel, A. S. Bracker, D. Gammon, C. Emary, and L. J. Sham, Phys. Rev. Lett. 99, 097401 (2007).

[25] E. B. Flagg, A. Muller, J. W. Robertson, S. Founta, D. G. Deppe, M. Xiao, W. Ma, G. J. Salamo, and C. K. Shih, Nat. Phys. 5, 203 (2009).

[26] D. Pinotsi and A. Imamoglu, Phys. Rev. Lett. 100, 093603 (2008).

[27] D. Press, T. D. Ladd, B. Zhang, and Y. Yamamoto, Nature (London) 456, 218 (2008).

[28] M. Palmer da Silva, arXiv:quant-ph/0405112. 